\title{
Acknowledgement to Reviewers for 2018
}

๑) Springer Nature B.V. 2019

Please join us in thanking all those scientists and experts in different fields represented in Environmental Fluid Mechanics for devoting time and effort to review the papers voluntarily. The Editor-in-Chief and publisher acknowledge the colleagues listed below for their excellent reviews of papers for which final decisions have been made during the period January 2018-December 2018.

Maryam Abdolahpour

Hassan Abdulmouti

J. Aberle

E. Adams

Claudia Adduce

Mohammad Afzali

Yulia Akutina

Julie Albagnac

Guttorm Alendal

Amir Abbas Aliabadi

Andrea Amicarelli

Naveed Anjum

David Apsley

S. Armfield

Abolfazl Aslani-Kordkandi

Joseph F. Atkinson

Hazi Azamathulla

Abul Baki

Gabriella Balacco

Ram Balachandar

Reza Barati

Sukanta Basu

Mouldi Ben Meftah

David Benson

Umberto Berardi

Bert Blocken

Alistair Borthwick

Michel Boufadel

Elie Bou-Zeid

Alan Brandt

Paul Branson

Hector Bravo
Michael Brown

Riccardo Buccolieri

Ralph Budwig

Daniel B. Bung

H. Burridge

Daniel Calvete

Rene A. Camacho

Francisco

Cantero-Chinchilla

Zhixian Cao

Pierre Carlotti

Jan Carmeliet

Luciano Castillo

Ian Castro

Oscar Castro-Orgaz

Daniela Cava

Jose Rafael Cavalcanti

Daniel

Caviedes-Vouillieme

Marcelo Chamecki

Tree Shu Ning Chan

Andy Chan

I-Chi Chan

Kuang-An Chang

Rutuja Chavan

Falin Chen

Hong Cheng

Lup Wai Chew

Yee Meng Chiew

Aaron Chow

Vincent Chu

Vladislava Churuksaeva
Omduth Coceal

Jordi Colomer

George Constantinescu

Benoit Cushman-Roisin

Alan Cuthbertson

Stuart Dalziel

Mauro De Marchis

Stephan de Wekker

Koustuv Debnath

Boudewijn Decrop

Eric Deleersnijder

Alex Demetracopoulos

Marco Dentz

Martin Detert

Subhasish Dey

Peter Diamessis

Nicholas Dodd

Job Dronkers

George Efthimiou

T. I. Eldho

Alessandro Errico

Vahid Etminan

Stefania Evangelista

Frederic Evers

Xing Fang

Duncan Farrow

Andrea Fenocchi

Domenico Ferraro

Almerindo Ferreira

Enrico Ferrero

Jan Bert Flor

Morris Flynn 


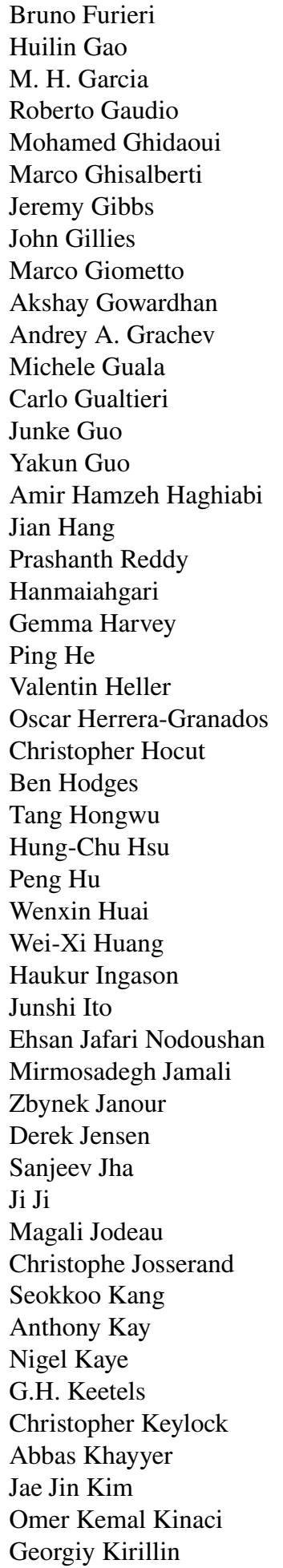

Hyeyun $\mathrm{Ku}$

Aytaç Kubilay

Bimlesh Kumar

Snehasis Kundu

Adrian Lai

Chris Lai

K. M. Lam

Gregory Lane-Serff

Javier Lara

B. Laval

Werner Lazeroms

Wei-Koon Lee

Quinn Lewis

Shuo Li

Yuguo Li

Alex Liberzon

Shao Ting J. Lien

Binliang Lin

Ying-Tien Lin

Chao Liu

Jianlin Liu

P. Liu

XiaoGuang Liu

Amparo Lopez-Jimenez

Iossif Lozovatsky

Jun $\mathrm{Lu}$

Daniel Lynch

L. R. M. Maas

Leo Maas

Madan Mahanty

Daniela Malcangio

Xi Mao

Corey Markfort

Matias Duran Matute

Talea Mayo

Igor Medvedev

Charles Melching

Yucong Miao

Emmanuel Mignot

Gretchen Miller

Abdolmajid

Mohammadian

Damith Mohotti

Ignacio Moncho-Esteve

Angel Monsalve

Paolo Monti

Michele Mossa

Mohamed Nabi

Daniel Nadeau
Negin Nazarian

C. O. $\mathrm{Ng}$

Vladimir Nikora

Roger Nokes

Pablo Ouro Barba

Manish Pandey

Ilias Papakonstantis

Panos N. Papanicolaou

Yong Sung Park

Hyoungsu Park

Frank Peeters

Efim Pelinovsky

Yann Peltier

David Plew

Suhas Pol

Marko Princevac

Panayotis Prinos

Sebastian Proust

Jaan Hui Pu

Lorenzo Raffaele

Majid Rahimpour

Chris Rehmann

Jonothan Remo

Jeffrey Rominger

Gabriel Rooney

Gerardo Ruiz

Mir-Jafar-Sadegh Safari

Francisco Salamanca

Julio Salcedo-Castro

Scott Salesky

Mats Sandberg

Michio Sanjou

Jose' Luis Santiago

Sankar Sarkar

Theresa Serra

Morteza Shakeri Majd

Ashish Sharma

John Z. Shi

WuRong Shih

James Shucksmith

Stefano Sibilla

Andrew Sleigh

Hassan Smaoui

Barton Smith

Sandra Soares-Frazão

Scott Socolofsky

Luca Solari

Julio Soria

Michael Spall 


$\begin{array}{lll}\text { Anastasios I. Stamou } & \text { Roberto Verzicco } & \text { Tiao-Jian Xu } \\ \text { Peter Stansby } & \text { Cara Walters } & \text { Weilin Xu } \\ \text { Ivana Stiperski } & \text { Benlong Wang } & \text { Xu-Feng Yan } \\ \text { Hongwu Tang } & \text { Chau Chang Wang } & \text { J. Q. Yang } \\ \text { Donatella Termini } & \text { Hang Wang } & \text { Elowyn Yarger } \\ \text { Firat Testik } & \text { Harry V. Wang } & \text { Xiping Yu } \\ \text { Francis Ting } & \text { Zhihua Wang } & \text { Dragan Zajic } \\ \text { Ali Tohidi } & \text { Ya Ping Wang } & \text { Dino Zardi } \\ \text { Talia Tokyay } & \text { Yongqi Wang } & \text { Luis Zavala Sanson } \\ \text { Daniela Tordella } & \text { Jeff Weil } & \text { Peter Zavialov } \\ \text { Desiree Tullos } & \text { Matthew Wells } & \text { Cheng Zeng } \\ \text { Wim Uijttewaal } & \text { Colin Whittaker } & \text { Huiming Zhang } \\ \text { Hugo Ulloa } & \text { Adam Witt } & \text { Ming Liang Zhang } \\ \text { Marius Ungarish } & \text { Ching-Sen Wu } & \text { Wei Zhang } \\ \text { GertJan van Heijst } & \text { Peng Wu } & \text { Yang Zhang } \\ \text { Cees van Rhee } & \text { Teng Wu } & \text { Lin Zhao } \\ \text { J. Vassilicos } & \text { Weiming Wu } & \text { Shiyuan Zhong } \\ \text { Kaisa Västilä } & \text { Alfred Wuest } & \text { Gongbo Zu } \\ \text { Subhas Venayagamoorthy } & \text { Davide Wüthrich } & \end{array}$

We apologize for any errors or inadvertent omissions.

Publisher's Note Springer Nature remains neutral with regard to jurisdictional claims in published maps and institutional affiliations. 